Anuario Latinoamericano Ciencias Políticas

y Relaciones Internacionales

vol. 10,2020

pp. $15-37$

\section{La diplomacia china hacia América Latina: bases cooperativas para la iniciativa de la Franja y la Ruta}

\section{Chinese Diplomacy Towards Latin American Countries. Cooperative Baseline for the Belt and Road Initiative}

\author{
Mariano Mosquera* \\ UNIVERSIDAD CATÓLICA DE CÓRDOBA \\ ARGENTINA \\ $\triangle$ mariano.mosquera@ucc.edu.ar \\ https://orcid.org/0000-0002-9037-545x
}

\title{
RESUMEN
}

En el año 2008 China difundió el Libro Blanco hacia América Latina, sentando las bases políticas de la cooperación omnidireccional con sus dos dimensiones: múltiples áreas de cooperación y aplicación diferenciada por país acorde a sus particularidades. Por otro lado, en el año 2013 China comienza a difundir la iniciativa de la Franja y la Ruta. En este contexto, nos preguntamos: ¿qué bases cooperativas se pueden identificar para la integración de América Latina en la iniciativa de la Franja y la Ruta? El estudio utilizó las categorías del Libro Blanco para analizar las comunicaciones del Ministerio de Relaciones Exteriores de la República Popular China hacia los países de América Latina entre los años 2008 y 2016. El método integró tres enfoques: estudios de agenda, análisis de contenido y análisis semántico-léxico. Como principal resultado, el estudio sobre la agenda diplomática de China hacia América Latina identificó múltiples áreas de cooperación, pero con una aplicación uniforme hacia los países de América Latina. Esto permite pensar en nuevas oportunidades para América Latina y, al mismo tiempo, en desafíos de diferenciación para el intercambio cooperativo en el marco del proyecto de la Franja y la Ruta.

PALABRAS CLAVE: iniciativa de la Franja y la Ruta, diplomacia, China, América Latina, omnidireccionalidad.

\footnotetext{
* Doctor en Ciencia Política de la Universidad Nacional de Córdoba y experto en Programación de la Universidad Tecnológica Nacional en Argentina. Director del Centro de Estudios La Franja y la Ruta de la Universidad Católica de Córdoba en Argentina. Fue profesor e investigador en la Universidad Sun Yat-sen en la República Popular China y becario de investigación en la Universidad de Harvard. Sus líneas de investigación son: discurso político chino, análisis institucional y procesamiento de lenguaje natural.
} 
Dossier América Latina: la iniciativa china de la Franja y la Ruta

\section{ABSTRACT}

In 2008, China disseminated the White Paper to Latin American countries, laying the political foundations of omnidirectional cooperation with its two dimensions multiple areas of cooperation and differentiated application by country according to its particularities. On the other hand, in 2013 China begins to spread the Belt and Road Initiative. In this context, our research question is: what cooperative baseline can be identified for the integration of Latin American region in the Belt and Road Initiative? The study used the categories of the White Paper to analyze communications of the Ministry of Foreign Affairs of the People's Republic of China to the Latin American countries between 2008 and 2016. The method integrated three approaches: agenda studies, content analysis and semantic-lexical analysis. As the main research result, the study on China's diplomatic agenda towards Latin American countries identified multiple areas of cooperation, but with uniform application to Latin American countries. This allows us to think about new exchange opportunities for Latin American region and, at the same time, about differentiation challenges for cooperative exchange within the framework of Belt and Road project.

KEYWORDS: Belt and Road Initiative, diplomacy, China, Latin America, omnidirectionality.

\section{Introducción}

La cooperación omnidireccional de China hacia los países de América Latina es definida, por primera vez, en el Documento sobre la Política de China hacia América Latina y el Caribe, de noviembre del año 2008 (DPCALC, 2008). Este documento político de la República Popular China, conocido como el Libro Blanco hacia América Latina, describe la omnidireccionalidad como una multiplicidad de áreas y subniveles de cooperación. Se trata, por lo tanto, de una perspectiva oficial china sobre la forma de entender la cooperación internacional hacia la región de América Latina.

A su vez, la cooperación omnidireccional supone una estrategia de aplicación diferenciada (Oviedo, 2006) según el contexto particular de cada país. El propio Libro Blanco dice: "Las naciones latinoamericanas y caribeñas exploran activamente los caminos de desarrollo ajustados a sus realidades nacionales." (DPCALC, 2008, p. 3). Este énfasis en la particularidad de la cooperación tiene un sustento político y teórico, ya que distintos principios políticos y de filosofía política china destacan a la cooperación como una dinámica de lo particular. Este es el caso de conceptos como el de pragmatismo político y el de armonía social, que son trabajados en el marco teórico de este trabajo. Ambos conceptos son fundamento para que nuestra hipótesis se defina a partir de la actuación de la cooperación como aplicación de la particularidad.

De esta forma, la hipótesis de este trabajo es que la agenda diplomática de China hacia América Latina se caracteriza por abarcar una multiplicidad 
de áreas de cooperación, que se diferencian según las particularidades de los países. Así, son dos los objetivos de esta investigación, donde cada uno se relaciona con cada una de las partes de nuestra formulación de la hipótesis. Los objetivos, desde la cooperación de China hacia América Latina, son: 1) determinar el grado de distribución de la cooperación en múltiples áreas y subniveles temáticos, 2) describir la cooperación según las diferencias entre países.

Estos objetivos nos permiten comprender las bases cooperativas entre China y América Latina -sus temas por países- y, por lo tanto, nos habilita a interpretar el punto de partida de oportunidades y desafíos para la integración de América Latina en la iniciativa de la Franja y la Ruta.

Para comprobar la hipótesis de investigación, se realiza un análisis de contenido sobre la agenda diplomática de China hacia los países de América Latina, entre el año 2008 y el año $2016^{1}$. La agenda diplomática es considerada un género específico (Dudley-Evans, 1996) que cumple las condiciones para el estudio de la iniciativa de cooperación omnidireccional desde el productor del discurso (Lyons, 1995).

Así, en el primer apartado de este trabajo se referencian antecedentes de estudio sobre la diplomacia china, destacando la originalidad de la presente investigación en ese marco. Los antecedentes contextualizan la discusión sobre oportunidades y riesgos en el marco de la iniciativa de la Franja y la Ruta, con la cual el presente trabajo concluye.

El segundo apartado define la cooperación omnidireccional desde sus dos dimensiones: multiplicidad y particularidad. Se parte de contextualizar las bases políticas de la cooperación de China hacia América Latina en el Libro Blanco y se construye, luego, la fundamentación política y teórica sobre la omnidireccionalidad. Se trata de un marco que define la orientación de los conceptos para el proceso de investigación. El tercer apartado desarrolla la metodología que combina estudios de agenda, análisis de contenido, y análisis semántico-léxico. Este apartado también define conceptos, pero en un sentido de utilidad operativa para la contrastación de la hipótesis de investigación. El cuarto apartado describe y analiza los resultados en términos del índice de variación cualitativa y de rangos de variabilidad entre países.

Finalmente, las conclusiones refieren a la comprobación parcial de nuestra hipótesis y discuten los desafíos - aprovechar oportunidades y disminuir riesgos- en la aplicación de la estrategia china de omnidireccionalidad hacia los países de América Latina, en el marco de la iniciativa de la Franja y la Ruta.

1 En 2016 surge el segundo Libro Blanco hacia América Latina, por lo tanto, el presente estudio se enfoca en la aplicación del primer Libro Blanco en todo su periodo de vigencia. Este lapso temporal coincide con el lanzamiento de la iniciativa de la Franja y la Ruta, de aquí su relevancia para esta investigación.
La diplomacia china hacia América Latina: bases cooperativas para la iniciativa de la Franja y la Ruta

Mariano Mosquera 
Dossier América Latina: la iniciativa china de la Franja y la Ruta

\section{Antecedentes}

Los estudios sobre la diplomacia china se caracterizan por una marcada diferenciación en etapas históricas, acorde a los cambios políticos producidos en ese país (Garner, 2008; Kurlantzick, 2007). A su vez, se trata de estudios que buscan especificar claramente el contexto de la relación diplomática, ya sea por país o por región, dados ciertos criterios (políticos o económicos) para acotar territorialmente el estudio (Hao, Wei y Dittmer, 2015). Existe una gran cantidad de estudios descriptivos sobre la diplomacia china basados en este esquema histórico-territorial.

Por otra parte, estudios más analíticos ponen el acento en la diplomacia como manifestación de "poder suave" (Nye Jr., 2004, p. 9), dentro de determinados marcos teóricos de relaciones internacionales, como la teoría de la interdependencia compleja (Keohane, 2006; Keohane y Nye Jr., 1989) o, de forma diferente, el enfoque constructivista (Vitelli, 2014).

De esta forma, con especificaciones de contexto y análisis de relaciones de poder, surgen estudios que caracterizan al ejercicio de la diplomacia china en términos positivos o negativos para con ciertos países o regiones (Avendaño Miranda, 2013; Lynch, 2013; Roy, 1996). También, ciertas investigaciones interpretan que la diplomacia china tiene el fin de proyectar una imagen específica al resto del mundo (Losada Olmos, 2013) por medio de su retórica (Corkin, 2014) e incluso sugieren la posibilidad de procesos hegemónicos mediados por el discurso (Beeson, 2009; Liebman, 2005; Roy, 1997). Aquí es importante resaltar, desde un punto de vista teórico, que toda orientación hegemónica de un discurso pone énfasis en la construcción de argumentos uniformes, distanciados de los "hechos", para satisfacer condiciones de control y reproducción (Davies, 2011).

En este contexto, una serie de estudios recientes sobre la diplomacia china han puesto su mirada en América Latina. Esto se debe al aumento de la actividad diplomática china en los últimos tiempos (Bonilla Soria y Herrera-Vinelli, 2020) y a una perspectiva sobre la función geopolítica de América Latina en el balance del poder mundial (Pastrana Buelvas y Vera, 2017). Estos estudios destacan el patrón positivo-negativo ya mencionado, pero en forma de oportunidades y riesgos de la relación entre China y América Latina (Salama, 2017; Terán Samanamud, 2014; Strauss y Armony, 2012; Lafargue, 2006).

Sin embargo, estos estudios, en la mayoría de los casos, no son de tipo empírico, salvo cuando se trata de investigaciones económicas como los estudios sobre comercio internacional o inversiones. Este último tipo de estudios es abundante y busca fundamentar con evidencia la relación económica entre China y América Latina (López Arévalo, Rodil Marzábal y Valdéz Gastelum, 2014; De Onis, 2014; Yaolin Wang, 2002).

En general, entonces, los estudios sobre la diplomacia china refieren a etapas históricas y son contextualizados por país o grupos de países. Lo importante a resaltar aquí es que esa diferenciación se produce bajo el supuesto de 
que cada contexto histórico-territorial representa cierta particularidad que merece un análisis específico (Shih, 2005). Luego, cuando se trata de estudios sobre la diplomacia china hacia América Latina el patrón destacado es el de oportunidades y riesgos, pero en general predominan los estudios que no son empíricos y las investigaciones económicas sobre el tema.

Desde el año 2014, en el marco de la iniciativa de la Franja y la Ruta, este patrón descrito en los estudios académicos se ha acentuado (Oliveira y Myers, 2020). En septiembre de 2013, el presidente chino Xi pronunció un discurso en la Universidad de Nazarbayev, Kazajstán, en el cual propuso construir "La Franja Económica de la Ruta de la Seda”, en sinergia con Asia Central. Luego, en octubre de ese mismo año, durante la Reunión de Líderes de $\mathrm{APEC}^{2}$, Xi planteó construir la "Ruta Marítima de la Seda del siglo XXI" con los países de la ASEAN ${ }^{3}$. Si bien la iniciativa de la Franja y la Ruta es un producto histórico que es extensión de la Ruta de la Seda antigua, actualmente adquiere un significado global con todas las regiones del mundo, incluida América Latina. De esta forma, en pocos años y desde comienzos de 2014, el proyecto ha pasado a la acción: China ha firmado una larga serie de acuerdos de cooperación con los países a lo largo de la Franja y la Ruta 4 .

Los estudios académicos sobre la iniciativa de la Franja y la Ruta definen objetivos claros para China: ampliar vías hacia el oeste para desarrollar regiones occidentales, estimular sectores industriales, abrir mercados para productos chinos, promover que otros países utilicen sus estándares tecnológicos y, a nivel general, extender su presencia e influencia a nivel internacional (Parra Pérez, 2018). Aunque la gran mayoría de autores aborda la iniciativa desde una perspectiva económica (Yong y Peiran, 2018; Wintgens, 2018; Lávut, 2018), es necesario abordar también su implicancia geopolítica (Acuña Ortigoza, Aguirre Saavedra, Ávila Ramírez y Mendoza Vera, 2018). La propuesta del proyecto de la Franja y la Ruta deja en evidencia la tensión y disputa por la gobernanza mundial que se da en el escenario internacional (Dirmoser, 2017). La gobernanza tiene características diversas con respecto a la hegemonía, tanto en su discurso y práctica, como en sus estrategias y sus proyectos de intervención (Margueliche, 2019). Para Dussel (2018), el proyecto de la Franja y la Ruta es una propuesta de globalización con características chinas y, como tal, es una narrativa que genera múltiples interrogantes y lecturas. Flint y Zhu (2019) plantean que China busca construir acuerdos que replican los mismos objetivos y formas que las potencias hegemónicas.

En definitiva, en el marco de la iniciativa de la Franja y la Ruta, se profundizan las lecturas económicas sobre la relación entre China y América Latina. Además, como riesgo, adquiere un mayor protagonismo la referencia a un dis-

\footnotetext{
2 Foro Multilateral de Cooperación Económica Asia-Pacífico, creado en 1989.

3 Asociación de Naciones del Sudeste Asiático, creado en 1967.

4 En el caso de América Latina, han firmado acuerdos relacionados a la iniciativa de la Franja y la Ruta: Brasil, Perú, Bolivia, Chile, Venezuela y Uruguay.
}

La diplomacia china hacia América Latina: bases cooperativas para la iniciativa de la Franja y la Ruta

Mariano Mosquera 
Dossier América Latina: la iniciativa china de la Franja y la Ruta curso de tipo hegemónico que implica un control externo sobre los patrones de desarrollo de América Latina (Chauvin y Fraser, 2019). Puede observarse que la hipótesis de nuestra investigación opera en un sentido contrario a los antecedentes de estudio descritos para la iniciativa de la Franja y la Ruta. Esto es así, ya que buscamos comprobar que China trabaja con un método cooperativo que enfatiza múltiples áreas de cooperación (no solamente se enfoca en la economía) y que diferencia su discurso según las particularidades de los países, lo cual contradice la forma de una narrativa hegemónica.

Este trabajo de investigación se centra en la dimensión política que determina la jerarquía de prioridades de las diversas áreas temáticas de cooperación. Se trata de un estudio de ciencia política, que abarca una época reciente (2008 a 2016) y que contextualiza territorialmente, pero, sobre todo, prueba la utilidad o no de esa contextualización por país. Además, con base en evidencia empírica, el presente estudio discute oportunidades y riesgos de la cooperación entre China y América Latina en el marco de la iniciativa de la Franja y la Ruta. Lo mencionado constituye la originalidad del presente trabajo en el contexto de los antecedentes que conforman el estado del arte en la materia.

\section{Marco teórico}

\section{Base política de la cooperación hacia América Latina}

En noviembre del año 2008 la República Popular China difundió el Documento sobre la Política de China hacia América Latina y el Caribe. Se trató del primer documento de este tipo dirigido exclusivamente a la región, sentando las bases políticas de las relaciones diplomáticas para un determinado periodo de tiempo. El propio Libro Blanco define su objetivo central como: "Manifestar con mayor claridad los objetivos de la política china hacia la región, plantear los principios rectores de la cooperación en las diversas áreas durante un determinado periodo del futuro y promover el continuo desarrollo sano, estable e integral de las relaciones sino-latinoamericanas y sino-caribeñas." (DPCALC, 2008, p. 8). En el Cuadro 1 se describen los principales apartados de este documento.

La cooperación omnidireccional ha sido introducida en el Documento sobre la Política de China hacia América Latina y el Caribe, específicamente en su parte IV. Este documento, nos dice que "se viene configurando un flamante panorama caracterizado por el desarrollo omnidireccional, multifacético y de múltiples niveles de las relaciones entre ambas partes." (DPCALC, 2008, p. 17).

El Libro Blanco hacia América Latina posee 4.674 tokens $^{5}$ mientras que el apartado IV posee 3.375 tokens, representando así un 72,2 \% del documento

5 Palabras o símbolos que constituyen la unidad mínima para los análisis automáticos de textos. 


\begin{tabular}{|c|c|}
\hline Apartado & Descripción \\
\hline Prólogo & $\begin{array}{l}\text { Describe un mundo en cambio, centrado en la multipolaridad } \\
\text { política y en la globalización económica. En este contexto, } \\
\text { destaca dos dimensiones centrales y un fin máximo para } \\
\text { cooperar con otros países: paz y desarrollo, para alcanzar un } \\
\text { mundo armonioso. Además, China propone una cooperación } \\
\text { basada en dos ejes: beneficio reciproco (o ganancia } \\
\text { compartida) y los "Cinco Principios de Coexistencia Pacífica"a. }\end{array}$ \\
\hline $\begin{array}{l}\text { Posición y Papel de } \\
\text { América Latina y el } \\
\text { Caribe (parte I) }\end{array}$ & $\begin{array}{l}\text { Destaca el potencial, como influencia internacional, de } \\
\text { América Latina con base en sus recursos naturales, estabilidad } \\
\text { política y crecimiento económico con impacto social. Esto } \\
\text { bajo las particularidades de cada país. }\end{array}$ \\
\hline $\begin{array}{l}\text { Relaciones entre China } \\
\text { y América Latina y el } \\
\text { Caribe (parte II) }\end{array}$ & $\begin{array}{l}\text { Se definen como relaciones entre países en una misma fase } \\
\text { de desarrollo. Luego el apartado resalta la historia de las } \\
\text { relaciones de China con América Latina: desde el año } 1949 \\
\text { contactos no gubernamentales, desde el año } 1970 \text { inicio de } \\
\text { relaciones diplomáticas con la mayoría de los países de la } \\
\text { región, desde el año } 2000 \text { contactos de alto nivel. }\end{array}$ \\
\hline $\begin{array}{l}\text { Política de China hacia } \\
\text { América Latina y el } \\
\text { Caribe (parte III) }\end{array}$ & $\begin{array}{l}\text { Destaca la altura estratégica de la cooperación con América } \\
\text { Latina según cuatro objetivos: } 1 \text {. Crear confianza y consensos, } \\
\text { basados en igualdad y respeto mutuo. 2. Cooperar basándose } \\
\text { en explorar ventajas y potencialidades, sobre todo en lo } \\
\text { comercial. 3. Extender la cooperación a otras áreas no } \\
\text { económicas. 4. Respetar el "Principio de una sola China”. }\end{array}$ \\
\hline $\begin{array}{l}\text { Fortalecimiento } \\
\text { de la Cooperación } \\
\text { Omnidireccional } \\
\text { entre China y América } \\
\text { Latina y el Caribe } \\
\text { (parte IV) }\end{array}$ & $\begin{array}{l}\text { Se trata de una descripción detallada de cuatro áreas de } \\
\text { cooperación (política; económica; cultural y social; de la paz, } \\
\text { la seguridad y la justicia) y sus múltiples subniveles por cada } \\
\text { área temática. En el anexo de este trabajo se describen con } \\
\text { detalle. }\end{array}$ \\
\hline $\begin{array}{l}\text { Relaciones entre China } \\
\text { y las Organizaciones } \\
\text { Regionales } \\
\text { Latinoamericanas } \\
\text { y Caribeñas (parte V) }\end{array}$ & $\begin{array}{l}\text { El apartado resalta la función de las organizaciones regionales } \\
\text { para la cooperación internacional. }\end{array}$ \\
\hline
\end{tabular}

a Se trata de cinco principios difundidos en el año 1954: respeto mutuo por la soberanía y la integridad territorial, no agresión mutua, no interferencia en los asuntos internos de otros países, igualdad y beneficio mutuo, coexistencia pacífica.

b Se trata del pedido de China para que los países se abstengan de desarrollar relaciones y contactos oficiales con Taiwán. En menor medida el principio incluye la causa del Tíbet.

Fuente: elaboración propia con base en el Documento sobre la Política de China hacia América Latina y el Caribe (DPCALC, 2008).
La diplomacia china hacia América Latina: bases cooperativas para la iniciativa de la Franja y la Ruta

Mariano Mosquera

\section{Cuadro 1.}

Descripción de apartados del Documento sobre la Política de China hacia América Latina y el Caribe (2008) 
Dossier América Latina: la iniciativa china de la Franja y la Ruta completo. La cooperación omnidireccional incluye cuatro áreas y múltiples subniveles por área (34 en total) que listamos en el anexo de este trabajo.

Adelantamos aquí que, a nivel metodológico, el presente trabajo analiza la cooperación omnidireccional en la agenda diplomática de China hacia América Latina considerando la clasificación por áreas temáticas y subniveles del Documento sobre la Política de China hacia América Latina y el Caribe del año 2008. Sin embargo, es importante - primero- observar que el Libro Blanco también otorga fundamento político y teórico sobre la omnidireccionalidad.

\section{Fundamento político y teórico de la omnidireccionalidad}

En este apartado desarrollamos el fundamento político y teórico de las dos dimensiones de la omnidireccionalidad: la multiplicidad y su aplicación basada en la particularidad.

Desde el punto de vista político, el Libro Blanco (DPCALC, 2008) describe a la omnidireccionalidad desde la multiplicidad y refiere a la aplicación particular por país en el apartado primero. Es decir, las bases políticas del Libro Blanco asumen un compromiso de respeto sobre la particularidad de los países de América Latina. Este último es el sentido dado a la utilización de la noción de pragmatismo político en la agenda diplomática de China hacia América Latina. Se trata de un concepto que busca evitar la uniformidad, el formalismo y luchar contra los consensos estables que condicionan de forma externa a los actores de la cooperación (Sanz, 2013; Dai, 2012; Huang, 2010; Li, 2002). Así, el pragmatismo chino implica considerar a los contextos particulares como fundamento de la toma de decisiones políticas (Xi, 2014). De esta forma, son recurrentes las menciones a la "cooperación pragmática" en la agenda diplomática de China hacia los países de América Latina y es, en muchos casos, indistinta su utilización con respecto al concepto de cooperación omnidireccional.

Por otra parte, desde el punto de vista de la filosofía política china, el Libro Blanco plantea como objetivo supremo el "promover la construcción de un mundo armonioso." (DPCALC, 2008, p. 10). Se trata ahora de un compromiso del Libro Blanco con el concepto de armonía social. Chenyang Li (2014) nos dice que las cinco características claves de la concepción confuciana de armonía son:

1. Heterogeneidad. La armonía presupone dos o más partes en coexistencia. Estas partes no son uniformes y poseen variadas disposiciones.

2. Tensión. Varias partes interactúan entre sí. La tensión en varios niveles surge naturalmente de la diferencia.

3. Coordinación y cooperación. Si bien la tensión puede resultar en conflicto, también establece restricciones a las partes en interacción y genera 
energía para avanzar hacia la coordinación. En coordinación las partes toleran al otro y preservan su particularidad.

4. Transformación y crecimiento. A través de la coordinación, la tensión es transformada y el conflicto es reconciliado en un entorno favorable para que cada parte prospere. En este proceso, las partes involucradas se someten a la mutua transformación y forman relaciones armoniosas.

5. Renovación. La armonía no es alcanzada como un estado final, sino como etapas de un proceso en marcha. Se admiten diferentes niveles. Una relación armoniosa se mantiene con la renovación continua. (Li, 2014, p. 9)

Los requerimientos para alcanzar la armonía social se asemejan notablemente a ciertas características de la cooperación en la teoría de juegos (Morgenstern, 1967). Especialmente, las condiciones de baja cantidad de jugadores y de relaciones no-mediadas en la interacción entre los jugadores (Li, 2006). Es decir, múltiples y particulares contextos, partes y procesos son condición para la armonía social como forma cooperativa de sociedad. Además, la noción de armonía social es central en la cultura política china (Li, 2006; Cheng, 1991) y ha sido revitalizada en el discurso político chino actual (Li, 2014; Choukroune y Garapon, 2007).

Entonces, la cooperación omnidireccional (en un sentido operativo), la cooperación pragmática (en un sentido político) y la cooperación armoniosa (en un sentido social ${ }^{6}$ ), pueden ser consideradas como distintos términos de aplicación de la lógica de la particularidad a la cooperación. En la agenda diplomática china es común que estos términos se utilicen indistintamente para reflejar el sentido chino de la cooperación y, además, el Libro Blanco hacia América Latina asume un compromiso político con estos términos.

En definitiva, la lógica de la particularidad exige multiplicidad de contextos de intervención y la multiplicidad de contextos requiere de tratamientos particulares, lo que significa que multiplicidad y particularidad se encuentran indefectiblemente vinculados en la política y filosofía china.

Entonces, decimos que la cooperación omnidireccional refiere a múltiples áreas y niveles en los cuales China busca cooperar con los países de América Latina. Pero se trata también de una iniciativa pragmática y de armonía asumida desde sus bases políticas. Se espera, entonces, que para cada país se defina una particular relación con China, otorgando distinto "peso" a cada área y cada subnivel temático de cooperación.

De esta forma, se justifica la definición de hipótesis de este trabajo, donde consideramos que la agenda diplomática de China hacia América Latina se caracteriza por abarcar una multiplicidad de áreas y subniveles de cooperación, que se diferencian según las particularidades de los países.
La diplomacia china hacia América Latina: bases cooperativas para la iniciativa de la Franja y la Ruta

Mariano Mosquera 
Dossier América Latina: la iniciativa china de la Franja y la Ruta

\section{Metodología}

¿Podemos medir la estrategia de cooperación omnidireccional de China hacia los países de América Latina? Realizaremos una medición sobre la agenda diplomática cooperativa china, específicamente con diez (10) países de América Latina ${ }^{7}$, pero tendremos que considerar múltiples requerimientos metodológicos para realizar esta tarea. Estos requisitos son: la construcción de un corpus (McEnery y Wilson, 2001) con base en un género representativo y la construcción de un método de categorización.

Por otra parte, una multiplicidad de enfoques ha sido combinada para dar respuesta a los requerimientos metodológicos. En particular tres macro-enfoques se integraron en la investigación: estudios de agenda (Aruguete, 2015), análisis de contenido (Krippendorff, 1990), y análisis semántico-léxico (Sinclair, 1991).

\section{Construcción del corpus}

La agenda diplomática de China hacia los países de América Latina (tomando como unidad de contexto a las comunicaciones del Ministerio de Relaciones Exteriores de la República Popular $\mathrm{China}^{8}$ ) cumple la condición de un género específico, al identificarse un "propósito comunicativo del autor que gobierna las decisiones a nivel gramatical y léxico” (Dudley-Evans, 1996, p. 219).

De esta forma, la agenda diplomática -por medio de las comunicaciones oficiales- se vincula de forma directa con nuestros objetivos de medición sobre el discurso cooperativo omnidireccional de China. Nótese que el trabajo combina la noción de agenda (temas y atributos) con el marco discursivo de un género (Lyons, 1995).

Así, el corpus relevado posee comunicaciones en forma de entrevistas, discursos políticos, notas en medios periodísticos, entre otros formatos narrativos 9 . Consideramos las comunicaciones entre los años 2008 y 2016 dada la fuente de categorización. El corpus fue dividido en diez países y se alteraron secciones que afectaban la representatividad del país (menciones a otros países dentro de la comunicación de un país). Se trata de un corpus de 399 comunicaciones y de 180629 tokens finales, con unos 11083 tokens referidos a la cooperación omnidireccional $(6,4 \%$ del total datos, que representan las áreas y subniveles definidas como cooperación omnidireccional en el Libro Blanco de 2008).

7 Argentina, Bolivia, Brasil, Chile, Colombia, Costa Rica, Ecuador, México, Perú y Uruguay.

8 El Ministerio de Relaciones Exteriores de la República Popular China diferencia la diplomacia por país en los registros de sus comunicaciones oficiales: https://www.fmprc.gov.cn/esp/ gihdq/ldmz/

9 Las comunicaciones oficiales del Ministerio de Relaciones Exteriores de la República Popular China analizan y sintetizan, en muchos casos, los acuerdos y tratados con los países de América Latina. 


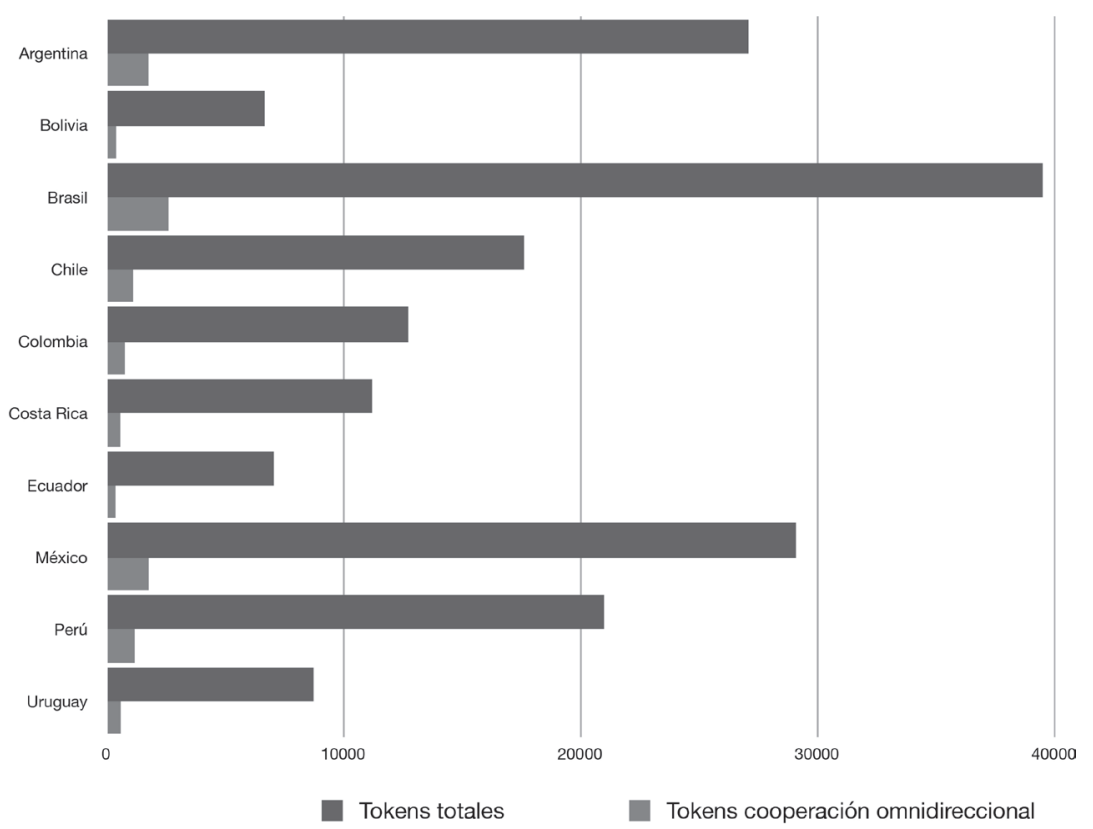

Fuente: elaboración propia con base en comunicaciones del Ministerio de Relaciones Exteriores de la República Popular China, 2008 a 2016.

\section{Categorización y análisis}

La categorización tiene el objeto de identificar términos clave (unidad de registro) en grupos, para que puedan ser analizados luego de aplicar un proceso de frecuencia léxica (Henderson y Hewings, 1990). El método busca que cada término justifique su inclusión exclusiva y de único principio clasificatorio en una categoría.

Respecto a la fuente de categorización de la cooperación omnidireccional, el Libro Blanco constituye la primera referencia en la construcción de las categorías que incluyen a los términos clave. Específicamente la Parte IV del Libro Blanco denominada Fortalecimiento de la Cooperación Omnidireccional entre China y América Latina y el Caribe contiene cuatro áreas de cooperación omnidireccional: 1) política; 2) económica; 3) cultural y social; 4) de la paz, la seguridad y la justicia. Cada una de estas áreas posee subniveles. Tanto las áreas como los subniveles representan las categorías de nuestro análisis, y el detalle de estos puede encontrarse en el anexo de este trabajo.

El Libro Blanco también define el horizonte temporal del corpus (periodo comprendido entre el 5 de noviembre de 2008 y el 24 de noviembre de 2016). El corte temporal se produce debido a que a fines del año 2016 China difundió el segundo Documento sobre la Política de China hacia América Latina y el Caribe.
La diplomacia china hacia América Latina: bases cooperativas para la iniciativa de la Franja y la Ruta

Mariano Mosquera

\section{Gráfico 1.}

Tokens en total de comunicaciones y en cooperación omnidireccional de China hacia países de América Latina (2008 a 2016) 
Dossier América Latina: la iniciativa china de la Franja y la Ruta
Debemos sumar, también, la identificación de "términos clave relacionados", identificados inductivamente (Rodríguez Sabiote, Lorenzo Quiles y Herrera Torres, 2005) desde el propio corpus y que pueden incluirse en las categorías previamente definidas por el Libro Blanco. Operaciones morfológicas, de "relación de sentido" (Lyons 1995, p. 81) y de contenido general-particular (Coulthard, 1996), fueron utilizadas para esta tarea. En total se utilizaron 23 reglas cualitativas, generales y particulares, de categorización.

Debe destacarse, particularmente, que por el enfoque de agenda de atributos (Ghanem, 2009) se identifican en el conteo (incidencia en la frecuencia léxica) los temas y atributos, así como los actores y mecanismos de relación entre actores, bajo la forma de términos cognitivos. Esta es una regla que se relaciona de forma directa con el marco general-particular del análisis de contenido (Coulthard, 1996).

Este tipo de diseño de investigación otorga como resultado final el posicionamiento relativo de ciertas categorías en el contexto cooperativo (y en un sentido cooperativo), pero no referencia de forma específica a la relación entre los términos (implicaría identificar verbos y conectores, por ejemplo). De hecho, el objeto de análisis semántico se refiere, en general, a sustantivos cognitivos (simples o compuestos en su relación entre las categorías) y adjetivos cognitivos (que caracterizan a sustantivos de la misma categoría o de otras categorías).

Por último, en esta tarea de categorización, es importante hacer una breve referencia sobre aquellos términos que no son parte de nuestras categorías. Es decir, términos que no son parte de nuestro nivel de anclaje (Samaja, 1996) en la construcción del dato. Esto en las comunicaciones del Ministerio de Relaciones Exteriores de la República Popular China se trata de un nivel abstracto que incluye cuatro ejes. Uno, temas y actores generales (no específicos de las categorías) y sus caracterizaciones. En este caso, se refiere a un discurso general sobre la cooperación para el desarrollo entre países, pero sin referencias concretas a las categorías. Además, dos, el nivel abstracto incluye a los denominados atributos afectivos (Ghanem, 2009), tres, a palabras vacías en el sentido del análisis de contenido (artículos, conectores, entre otros) y, cuatro, a palabras que no suman información en el sentido de las categorías (determinados nombres propios, por ejemplo).

Para realizar estas actividades mencionadas, se construyó con los términos clave un gazetteer (diccionario) que diferencia áreas y subniveles de cooperación, para ser corrido en el software libre GATE de la Universidad de Sheffield ${ }^{10}$. Esto es relevante, a su vez, al ser una aplicación para grandes volúmenes de datos (big data). El gazetteer final es un diccionario de 1123 términos simples y compuestos, dividido por áreas y subniveles de cooperación. El diccionario, al ser corrido en el software, identifica las frecuencias de cada categoría y por lo tanto se genera información sobre la distribución de las categorías. También, se aplicó un control de desambiguación (Sinclair, 1991) luego del conteo automático. Este

\footnotetext{
${ }^{10}$ GATE (Versión 8.4.1) [software]. (2017). URL: https://gate.ac.uk: Universidad de Sheffield.
} 
control busca identificar si el término en cuestión analizado es efectivamente parte de la categoría que lo está midiendo. Se encontraron casos de términos cuyo sentido no refería a un contexto cooperativo, términos cuyo sentido era de un nivel general-abstracto, términos compuestos en los cuáles se debió evitar el doble conteo, la repetición de términos claves entre paréntesis, y nombres propios de una categoría que incluían términos de otras categorías. Se elaboraron en este sentido ocho reglas de desambiguación. A su vez, el gazetteer fue modificado para reducir su margen de error, en los casos en que esto fue posible sin perder información de conteo. El nivel de error del diccionario es finalmente de $4,1 \%$, con un mejor funcionamiento para frecuencias absolutas altas. Todos los errores (474) fueron identificados con su número de token. Finalmente, se tomaron decisiones en cuanto a la selección del modelo de interpretación estadístico (Woods, Fletcher y Hughes, 1991) y se registraron sus correspondientes cálculos. Se generaron tablas con frecuencias absolutas y relativas, por áreas y subniveles, y se aplicó para la medición del grado de distribución el Î́ndice de Variación Cualitativa (IVC). Este índice posee la siguiente fórmula:

$$
I V C=\frac{1-\sum_{i=1}^{k} p_{i}^{2}}{(k-1) / k}
$$

Donde $k$ es el número de categorías de la variable (subniveles del área) y pi la frecuencia relativa asociada a cada una de ellas. El IVC expresa el grado en que los casos están dispersos en las diferentes categorías de la variable, alcanzando su máximo $(\mathrm{IVC}=1)$ en el caso en que las frecuencias relativas sean iguales para todas las categorías de la variable. El IVC será igual a 0 cuando la frecuencia relativa de una categoría de la variable fuese igual a 1 . El índice es adecuado para variables cualitativas, politómicas y nominales y utiliza las proporciones de corpus con distintos tamaños, lo cual lo hace compatible para nuestro análisis. Por otra parte, para observar diferencias entre países el estudio utilizó rangos inter-cuartiles, ya que estos son robustos ante valores extremos que pueden merecer una descripción particular.

\section{Resultados}

Este apartado describe y analiza los resultados de la investigación en tres secciones, diferenciadas por el tipo de hallazgo del estudio.

\section{Grado de distribución de las múltiples direcciones}

Se han identificado altos valores del Índice de Variación Cualitativa (IVC) entre áreas de cooperación, tal como puede observarse en la siguiente tabla.
La diplomacia china hacia América Latina: bases cooperativas para la iniciativa de la Franja y la Ruta

Mariano Mosquera 


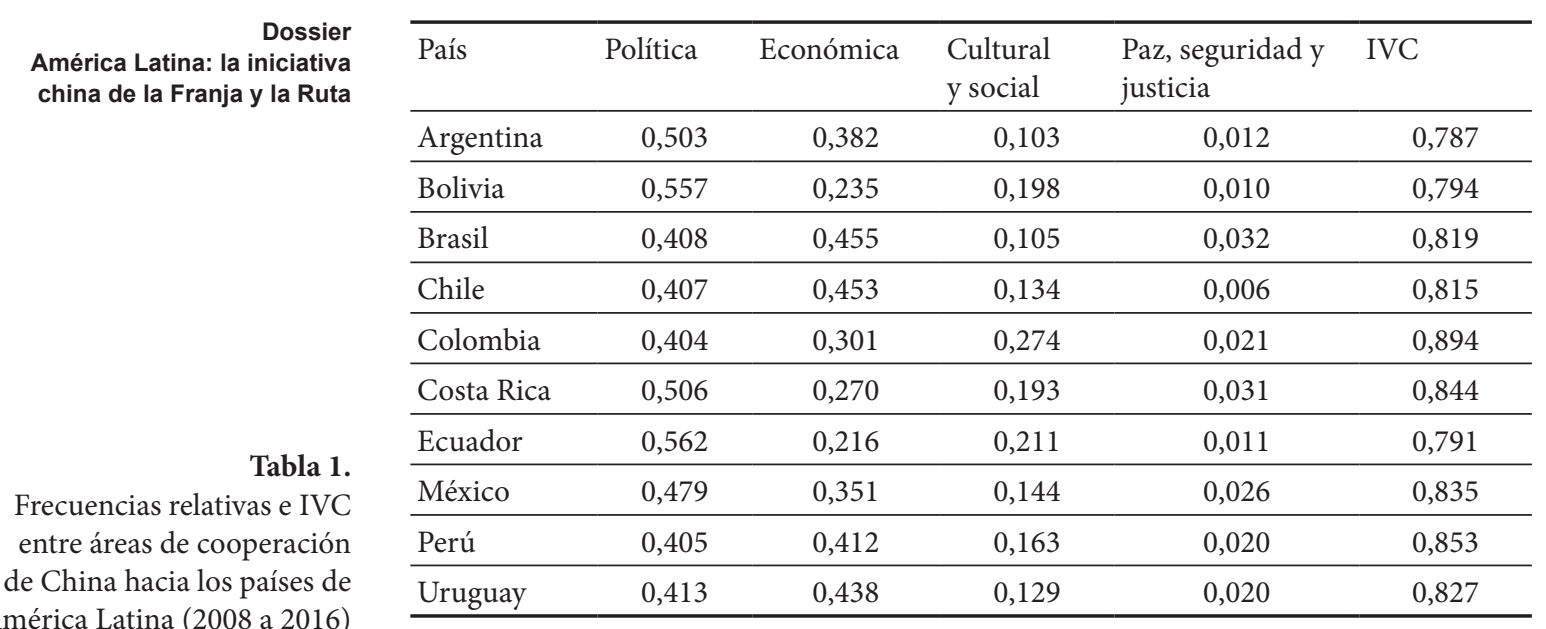

Fuente: elaboración propia con base en comunicaciones del Ministerio de Relaciones Exteriores de la República Popular China, 2008 a 2016.

También altos valores de IVC se han registrado entre los 14 subniveles del área económica, entre los 11 subniveles del área cultural y social, y entre los 6 subniveles del área política. En ese orden considerando el IVC de los diez países $(0,88 ; 0,80 ; \mathrm{y} 0,66$ de promedio respectivamente).

Hay valores menores de IVC entre los 3 subniveles de cooperación en el área de la paz, la seguridad y la justicia, pero esto se debe a sus bajas frecuencias absolutas.

De esta forma, la omnidireccionalidad entendida -en este apartado- como el grado de distribución de la cooperación en múltiples direcciones de cooperación de China hacia diez países de América Latina queda demostrada en este trabajo. Esto a partir del análisis de frecuencias léxicas de términos claves en categorías de cooperación previamente definidas por el productor del discurso de la agenda diplomática.

\section{Forma en la distribución de las múltiples direcciones}

Una estrategia de omnidireccionalidad, según la segunda parte de la formulación de nuestra hipótesis, genera condiciones para que con cada país se defina -según sus propias características particulares- el "peso" de cada área o de cada subnivel de cooperación, de cada tema o de cada relacionamiento con actores.

Sin embargo, a pesar de lo mencionado anteriormente, la investigación no puede describir mayores diferencias entre los países por área o subnivel temático de cooperación.

El IVC no nos proporciona información respecto a la forma que adopta la distribución de la cooperación, sino que solamente nos refiere al grado de dis- 
tribución que se tiene. Entonces, si bien se ha demostrado que la cooperación de China hacia los países de América Latina incluye múltiples direcciones, la forma de la distribución hallada en esta cooperación es muy uniforme entre países.

Esto es más representativo por subniveles que por áreas de cooperación. Los rangos inter-cuartiles, por subnivel, para el área política tienen un valor máximo de 0,099, mientras que para el área económica tienen un valor máximo de 0,064 y para el área cultural y social de 0,051 (con la única excepción del subnivel 2). El área de cooperación en la paz, la seguridad y la justicia posee mayores rangos inter-cuartiles, pero por sus bajas frecuencias absolutas. La forma dominante de la distribución del discurso de la cooperación de China hacia los países de América Latina, según la mediana entre países, puede verse en el siguiente gráfico.

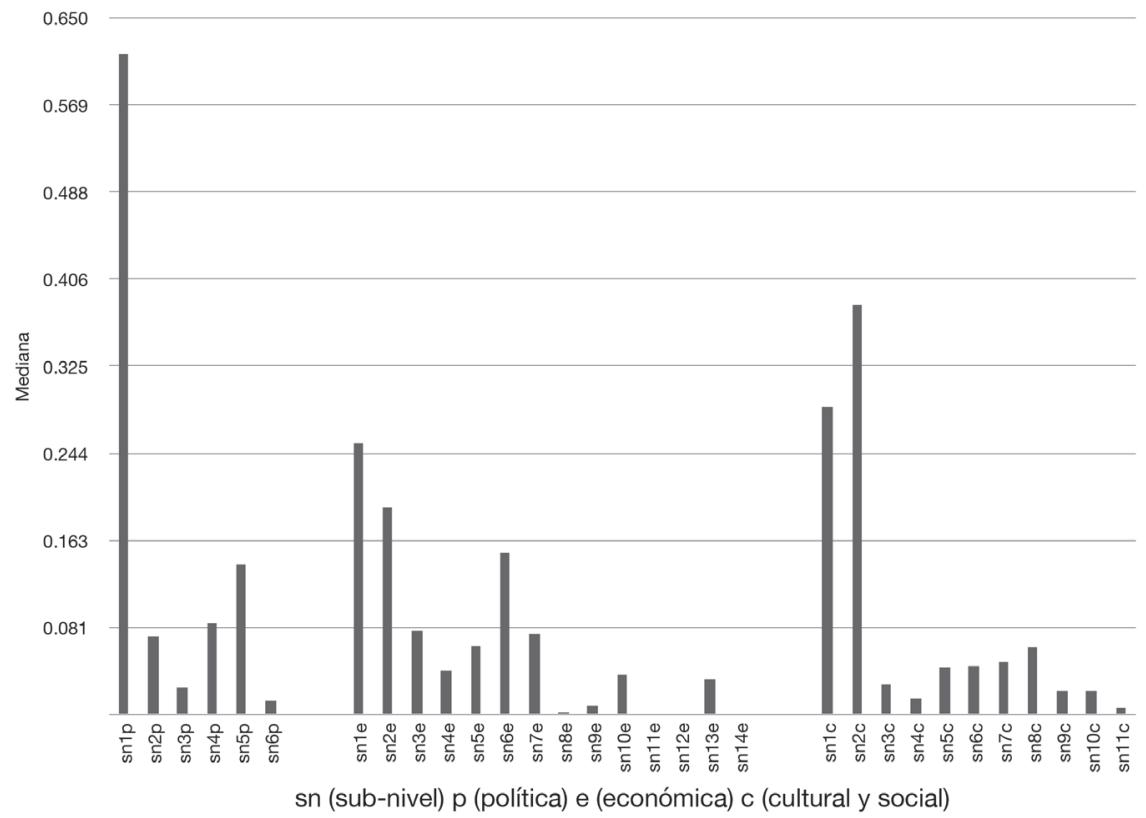

Fuente: elaboración propia con base en comunicaciones del Ministerio de Relaciones Exteriores de la República Popular China, 2008 a 2016.

Esto significa que China coopera con todos los países analizados en múltiples temas, pero en proporciones similares sin diferenciar condiciones particulares de los países.

La agenda política es, en general para todos los países, una agenda de cooperación de alto nivel ( $\mathrm{sn} 1 \mathrm{p}$ ) y de asuntos internacionales ( $\mathrm{sn} 5 \mathrm{p}$ ), con algún interés legislativo (sn2p), y escaso interés por la cooperación entre partidos políticos y con los gobiernos locales ( $s n 3 p$ y sn6p). Las excepciones a esta uniformidad que pueden mencionarse de la agenda política son: el caso de Brasil con una ma-
La diplomacia china hacia América Latina: bases cooperativas para la iniciativa de la Franja y la Ruta

Mariano Mosquera

\section{Gráfico 2.}

Uniformidad de la cooperación de China hacia los países de América Latina. Año 2008 a 2016 (según medianas de frecuencias relativas por subnivel de área) 
Dossier América Latina: la iniciativa china de la Franja y la Ruta yor cooperación ${ }^{11}$ en asuntos internacionales con China, dada su convergencia en el grupo de países BRICS ${ }^{12}$; un mayor intercambio entre partidos políticos con Bolivia y con Ecuador que con el resto de los países; y, finalmente, una mayor cooperación entre gobiernos locales con México, este último caso debido, en general, a la cooperación de la ciudad de México con ciudades de China.

Por otra parte, la agenda económica es una agenda comercial (sn1e) y, luego, de inversión en infraestructura (sn2e y sn6e). En algunos casos (menos de la mitad del peso de la inversión) se referencia al financiamiento ( $\operatorname{sn} 3 \mathrm{e})$. Ya en menor nivel, hay interés por la cooperación en energías ( $s n 7 e$ ) y en industria (sn5e), y es todavía menor la cooperación en agricultura (sn4e) y turismo (sn10e). Particularmente como excepciones a la uniformidad del discurso económico se destacan: Chile y Costa Rica poseen una agenda comercial con valores extremos, superiores a la mediana; Brasil posee una agenda financiera e industrial muy elevada con China; y Argentina tiene una cooperación agrícola con China superior al resto de los países.

Por su parte, la agenda cultural y social es una agenda de alto valor para la cooperación científica, tecnológica y educativa (sn2c) y para la cooperación en cultura y deporte (sn1c). Los valores bajan considerablemente para la cooperación en cambio climático $(\operatorname{sn} 8 \mathrm{c}) \mathrm{y}$, luego, las medianas de frecuencias son muy bajas para la cooperación en protección del medio ambiente, la cooperación entre organizaciones civiles y la cooperación en prensa (sn7c, sn6c y sn $5 c$, respectivamente). Algunas excepciones se producen en esta área por casos muy particulares de cooperación: como una mayor cooperación científica con Bolivia por la cooperación con China en el lanzamiento del satélite boliviano TKSAT-1 y una mayor cooperación médica y sanitaria $(\operatorname{sn} 3 \mathrm{c}$ ) con México por el brote de influenza en el año 2009. Como otras excepciones a la uniformidad del área decimos que: Colombia se destaca con una mayor cooperación cultural (sn1c); Chile y Ecuador con una mayor cooperación en desastres naturales (sn10c); y Perú con una mayor cooperación en lucha contra la pobreza $(\operatorname{sn} 11 \mathrm{c})$ que el resto de los países.

La agenda cooperativa en la paz, seguridad y justica es muy baja en frecuencias absolutas. Sólo puede destacarse que México y Argentina poseen una mayor cooperación judicial que los otros países y que Brasil tiene una mayor cooperación en seguridad no tradicional que el resto de los países.

\section{Peso económico en la cooperación}

Por su parte, la forma de la distribución entre macro-áreas muestra a la cooperación cultural y social, y a la cooperación en la paz, seguridad y justicia siempre

11 No en términos absolutos, sino siempre en peso relativo dentro del área de cooperación correspondiente.

12 Organización política constituida por Brasil, Rusia, India, China y Sudáfrica en el año 2006. 
en tercer y cuarto lugar, respectivamente. En este caso sí resulta relevante que existe una mayor variabilidad entre países en la cooperación del área económica (con un rango inter-cuartil de 0,154 ). Esto se debe a una distribución más marcada de tres clases de países. Países con un mayor predominio económico en la agenda con China (Brasil, Chile y en menor medida Uruguay y Perú), países con baja importancia económica (Ecuador, Bolivia y en menor medida Costa Rica y Colombia) y países cerca de la mediana económica como Argentina y México.

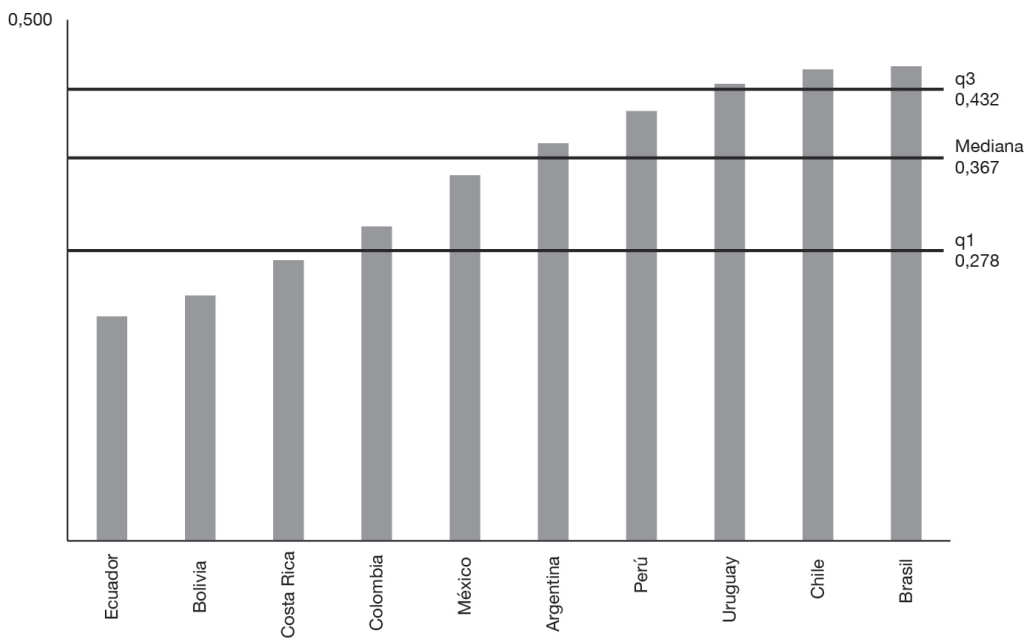

Fuente: elaboración propia con base en comunicaciones del Ministerio de Relaciones Exteriores de la República Popular China, 2008 a 2016.

Se destaca, particularmente, que sólo los primeros cuatro países tienen agendas económicas que superan las frecuencias de sus agendas políticas.

\section{Conclusiones}

Luego del proceso de investigación podemos afirmar los siguientes tres puntos. Primero, la cooperación omnidireccional de China con los países de América Latina, en la agenda diplomática, abarca una multiplicidad de áreas y subniveles, es decir, una multiplicidad de temas y de relacionamiento con actores. Segundo, la cooperación omnidireccional china con los diversos países de América Latina se aplica de forma uniforme, sin respetar la lógica de diferenciación particular por país. Tercero, en la cooperación omnidireccional de China hacia América Latina, solamente dos puntos parecen estar indicando una mayor orientación de particularidad por país: la presencia de algunas excepciones y la variabilidad de la cooperación económica. Sin embargo, esta última solamente cuando se considera al área económica en un nivel macro,
La diplomacia china hacia América Latina: bases cooperativas para la iniciativa de la Franja y la Ruta

Mariano Mosquera

\section{Gráfico 3.}

Frecuencia relativa de la cooperación económica en relación con la cooperación omnidireccional de China hacia los países de América Latina. Identificación de mediana y cuartiles (2008 a 2016) 
Dossier América Latina: la iniciativa china de la Franja y la Ruta ya que internamente el discurso cooperativo económico repite los patrones de uniformidad. De esta forma, la hipótesis de este trabajo sólo se comprueba parcialmente, aceptando la primera parte de su formulación, pero no probando la segunda parte.

Como discusión expresamos las siguientes interpretaciones sobre oportunidad y riesgo en la cooperación entre China y América Latina en el marco de la iniciativa de la Franja y la Ruta.

La cooperación omnidireccional puede ser una oportunidad para el intercambio en múltiples áreas, evitando una relación entre China y América Latina centrada únicamente en el comercio. De esta forma, observar la iniciativa de la Franja y la Ruta como un proyecto de comercio e inversiones representa una mirada restrictiva que no se corresponde con las bases cooperativas de la diplomacia de China hacia la región de América Latina.

Como riesgo, la cooperación omnidireccional china puede estar representando una contradicción entre los fundamentos de la omnidireccionalidad y su aplicación efectiva, adquiriendo forma de discurso hegemónico, dada la distribución uniforme hallada en esta investigación ${ }^{13}$. Es posible que estadios iniciales de adaptabilidad del discurso puedan justificar políticamente la uniformidad de la cooperación omnidireccional o, por otra parte, que otros estudios centrados en la acción de la cooperación puedan contradecir este trabajo con nueva evidencia empírica. Sin embargo, a nivel discursivo, en la agenda diplomática con América Latina, la presencia de uniformidad es concreta en esta investigación. En este sentido, una integración de América Latina en la iniciativa de la Franja y la Ruta debe contemplar la diferenciación de las particularidades de los países. Esto resulta importante para realizar las máximas de la orientación cooperativa China, pero también para evitar discursos que diluyan las particularidades y fomenten la reproducción y el control externo sobre los patrones de desarrollo de América Latina.

13 La discusión sobre antecedentes teóricos en este trabajo refleja la relación entre retórica y hegemonía. En este caso la distribución del discurso puede indicar una "limitación-compartimentación de la acción y su correspondiente legitimación de relaciones de poder" (Stoddart, 2007, p. 209). 


\section{Anexo. Áreas y subniveles de cooperación omnidireccional según Documento sobre la Política de China hacia América Latina y el Caribe (2008)}

\begin{tabular}{|c|c|}
\hline Área & Subnivel (sn) \\
\hline \multirow[t]{6}{*}{ Política } & 1. Intercambio de alto nivel \\
\hline & 2. Intercambio entre los órganos legislativos \\
\hline & 3. Intercambio entre los partidos políticos \\
\hline & 4. Mecanismos de consulta \\
\hline & 5. Cooperación en asuntos internacionales \\
\hline & 6. Contactos entre los gobiernos locales \\
\hline \multirow[t]{14}{*}{ Económica } & 1. Comercio \\
\hline & 2. Cooperación en inversión \\
\hline & 3. Cooperación financiera \\
\hline & 4. Cooperación agrícola \\
\hline & 5. Cooperación industrial \\
\hline & 6. Construcción de infraestructuras \\
\hline & 7. Cooperación en recursos y energías \\
\hline & 8. Cooperación aduanera \\
\hline & 9. Cooperación en inspección de calidad y cuarentena \\
\hline & 10. Cooperación turística \\
\hline & 11. Reducción y condonación de deudas \\
\hline & 12. Asistencia económica y técnica \\
\hline & 13. Cooperación multilateral \\
\hline & 14. Cooperación entre las cámaras y promotoras de comercio \\
\hline \multirow[t]{11}{*}{ Cultural y social } & 1. Intercambio cultural y deportivo \\
\hline & 2. Cooperación científica, tecnológica y educativa \\
\hline & 3. Cooperación médica y sanitaria \\
\hline & 4. Cooperación consular e intercambio de personal \\
\hline & 5. Cooperación en prensa \\
\hline & 6. Intercambio entre los pueblos \\
\hline & 7. Cooperación en la protección del medio ambiente \\
\hline & 8. Cooperación contra el cambio climático \\
\hline & 9. Cooperación en recursos humanos y seguridad social \\
\hline & $\begin{array}{l}\text { 10. Reducción de calamidades, socorro a damnificados } \\
\text { y asistencia humanitaria }\end{array}$ \\
\hline & 11. Cooperación en el alivio de la pobreza \\
\hline \multirow{3}{*}{$\begin{array}{l}\text { La paz, la seguridad } \\
\text { y la justicia }\end{array}$} & 1. Intercambio y colaboración militares \\
\hline & 2. Cooperación judicial y policial \\
\hline & 3. Seguridad no tradicional \\
\hline
\end{tabular}

Fuente: elaboración propia con base en el Documento sobre la Política de China hacia América Latina y el Caribe (DPCALC, 2008).
La diplomacia china hacia América Latina: bases cooperativas para la iniciativa de la Franja y la Ruta

Mariano Mosquera 
Dossier América Latina: la iniciativa china de la Franja y la Ruta

\section{Referencias}

Acuña Ortigoza, M., Aguirre Saavedra, E., Ávila Ramírez, P. y Mendoza Vera, A. (2018). Ruta de la Seda: Nuevas alianzas para la participación de América Latina. Revista Venezolana de Gerencia, vol. 23, no. 83, pp. 530-542.

Aruguete, N. (2015). El Poder de la Agenda. Política, Medios y Público. Buenos Aires: Biblos. Avendaño M., Lilliana L. (2013). Del aprendizaje a la globalización: la influencia del mercado y las instituciones en las multinacionales chinas. Investigación Económica, vol. 72, no. 285, pp. 3-39. http://dx.doi.org/10.1016/S0185-1667(13)72595-5

Beeson, M. (2009). Hegemonic Transition in East Asia? The Dynamics of Chinese and American Power. Review of International Studies, vol. 35, no. 1, pp. 95-112. http://dx.doi. org/10.1017/S0260210509008341

Bonilla Soria, A. y Herrera-Vinelli, L. (2020). CELAC como vehículo estratégico de relacionamiento de China hacia América Latina (2011-2018). Revista CIDOB d' Afers Internacionals, no. 124, pp. 173-198. https://doi.org/10.24241/rcai.2020.124.1.173

Chauvin, L. y Fraser, B. (2019). South America is Embracing Beijing's Science Silk Road. Nature, China's Science Silk Road, part 4. Recuperado el 8 de junio de 2020, de https:// www.nature.com/immersive/d41586-019-01127-4/index.html

Cheng, C. (1991). New Dimensions of Confucian and Neo-Confucian Philosophy. Albany: State University of New York Press.

Choukroune, L. y Garapon, A. (2007). The Norms of Chinese Harmony: Disciplinary Rules as Social Stabilizer. China Perspectives, vol.71, no. 3, pp. 36-49. Recuperado el 8 de junio de 2020, de http://www.jstor.org/stable/24053278

Corkin, L. J. (2014). China's Rising Soft Power: The Role of Rhetoric in Constructing China-Africa Relations. Revista Brasileira de Política Internacional, no. 57, pp. 49-72. http://dx.doi.org/10.1590/0034-7329201400204

Coulthard, M. (1996). On Analysing and Evaluating Written Text. En M. Coulthard (Ed.), Advances in Written Text Analysis (pp. 219-228). London: Routledge.

Dai, B. (2012). Stick to the Path of Peaceful Development. Societas Et Res Publica, vol. 1, no. 2, pp. 15-29.

Davies, J. S. (2011). Challenging Governance Theory: from Networks to Hegemony. London: Policy Press.

De Onis, J. (2014). China's Latin Connection: Eclipsing the US? World Affairs, vol. 176, no. 5, pp. 62-68. Recuperado el 8 de junio de 2020, de http://www.jstor.org/stable/43555053

Dirmoser, D. (2017). La gran marcha china hacia el oeste. El megaproyecto de la nueva Ruta de la Seda. Nueva Sociedad, no. 270, pp. 1-11.

DPCALC (2008). Documento sobre la Política de China hacia América Latina y el Caribe, del Gobierno de la República Popular China, con fecha del 5 de noviembre de 2008.

Dudley-Evans, A. (1996). Genre Analysis: An Approach to Text Analysis for ESP. En M. Coulthard (Ed.), Advances in Written Text Analysis (pp. 219-228). London: Routledge.

Dussel, E. (2018). La Iniciativa La Franja y la Ruta en América Latina: facilitación del comercio de América Latina y el Caribe con China. En J. Shixue y F. Mallimaci (Eds.), La Franja y la Ruta: Iniciativa China de Cooperación con América Latina y el Caribe (pp. 141-146). Buenos Aires: Ediciones UNTDF. 
Flint, C. y Zhu, C. (2019). The geopolitics of connectivity, cooperation, and hegemonic competition: The Belt and Road Initiative. Geoforum, vol. 99, pp. 95-101. https://doi. org/10.1016/j.geoforum.2018.12.008

Garner, R. E. (2008). Understanding Contemporary China. Boulder: Lynne Rienner.

Ghanem, S. (2009). Filling in the Tapestry: The Second Level of Agenda Setting. En M. McCombs, D. L. Shaw y D. Weaver (Eds.), Communication and Democracy. Exploring the Intellectual Frontiers in Agenda Setting Theory (pp. 3-14). Mahwah: Lawrence Erlbaum.

Hao, Y., Wei, G. y Dittmer, L. (2015). Challenges to Chinese Foreign Policy: Diplomacy, Globalization and the Next World Power. Lexington: The University Press of Kentucky.

Henderson, W. y Hewings, A. (1990). Language and Model Building? En A. Dudley-Evans y W. Henderson (Eds.), The Language of Economics: The Analysis of Economics Discourse (pp. 43-54). London: Macmillan \& British Council.

Huang, Y. (2010). Debating China's Economic Growth: The Beijing Consensus or The Washington Consensus. Academy of Management Perspectives, vol. 24, no. 2, pp. 31-47. Recuperado el 8 de junio de 2020, de http://www.jstor.org/stable/25682397

Keohane, R. y Nye Jr., J. S. (1989). Power and Interdependence: World Politics in Transition. Boston: Brown, Little and Company.

Keohane, R. (2006). Liberalism, Power and Governance in a Partially Globalized World. Beijing: Peking University Press.

Krippendorff, K. (1990). Metodología de análisis de contenido. Teoría y práctica. Barcelona: Paidós Comunicación.

Kurlantzick, J. (2007). China's New Diplomacy and Its Impact on the World. The Brown Journal of World Affairs, vol. 14, no. 1, pp. 221-235. Recuperado el 8 de junio de 2020, de http://www.jstor.org/stable/24590703

Lafargue, F. 2006. La Chine en Amérique latine: Stratégies, objectifs et limites d'une présence. Perspectives Chinoises, no. 97, pp. 2-11. Recuperado el 8 de junio de 2020, de http://www.jstor.org/stable/24071647

Lávut, A. (2018). La iniciativa china "La Franja y la Ruta” y los países de América Latina y el Caribe. Iberoamérica Journal, no. 2, pp. 42-67.

Li, C. (2006). The Confucian Ideal of Harmony. Philosophy East and West, vol. 56, no. 4, pp. 583-603. Recuperado el 8 de junio de 2020, de http://www.jstor.org/stable/4488054

Li, C. (2014). The Confucian Philosophy of Harmony. New York: Routledge.

Li, L. (2002). Research on China’s Seeking-No-Hegemonism Thought. Tesis de postgrado. Sun Yat-sen University, la República Popular China.

Liebman, A. (2005). Trickle-down Hegemony? China’s "Peaceful Rise" and Dam Building on the Mekong. Contemporary Southeast Asia, vol. 27, no. 2, pp. 281-304. Recuperado el 8 de junio de 2020, de http://www.jstor.org/stable/25798737.

López Arévalo, J. A., Rodil Marzábal, Ó. y Valdéz Gastelum, S. (2014). La irrupción de China en el TLCAN: efectos sobre el comercio intra-industrial de México. Economía UNAM, vol. 11, no. 31, pp. 84-113. http://dx.doi.org/10.1016/S1665-952X(14)70446-3

Losada Olmos, I. (2013). La diplomacia pública de China en América Latina. Lecciones para Chile. En I. Rodríguez Aranda y S. Yang (Eds.), Gestión y Análisis de Políticas Públicas. Santiago de Chile: RIL. Recuperado el 8 de junio de 2020, de http://www.redalyc.org/ articulo.oa?id=281532956009
La diplomacia china hacia América Latina: bases cooperativas para la iniciativa de la Franja y la Ruta

Mariano Mosquera 
Dossier América Latina: la iniciativa china de la Franja y la Ruta
Lynch, D. C. (2013). Securitizing Culture in Chinese Foreign Policy Debates. Implications for Interpreting. Asian Survey, vol. 53, no. 4, pp. 629-652. Recuperado el 8 de junio de 2020, de http://www.jstor.org/stable/10.1525/as.2013.53.4.629

Lyons, J. (1995). Linguistic Semantics. An Introduction. Cambridge: Cambridge University Press.

McEnery, A. y Wilson, A. (2001). Corpus Linguistics. Edinburgh: Edinburgh University Press.

Margueliche, J. C. (2019). La nueva Ruta de la Seda china y sus implicancias espaciales. Un análisis teórico desde la perspectiva de la (des) territorialización. En L. Bogado Bordazar, M. Staiano y M. Caubet (Eds.), China y una nueva estrategia geopolítica y global. La Iniciativa de La Franja y la Ruta (pp. 96-111). La Plata: Instituto de Relaciones Internacionales y Centro de Estudios Chinos de la UNLP.

Morgenstern, O. (1967). Game Theory: A New Paradigm of Social Science. En F. Zwicky y A. G. Wilson (Eds.), New Methods of Thought and Procedure (pp. 203-227). Berlin: Springer.

Nye Jr., J. S. (2004). Soft Power: The Means to Success in World Politics. New York: Public Affairs.

Oliveira, G. y Myers, M. (2020). The Tenuous Co-Production of China's Belt and Road Initiative in Brazil and Latin America. Journal of Contemporary China, ahead of print, pp. 1-19. https://doi.org/10.1080/10670564.2020.1827358

Oviedo, D. (2006). China: visión y práctica de sus llamadas relaciones estratégicas. Estudios de Asia y África, vol. 41, no. 3(131), pp. 385-404.

Parra Pérez, Á. (2018). El juego geopolítico de la nueva Ruta de la Seda en Asia. Instituto Español de Estudios Estratégicos, no. 126.

Pastrana Buelvas, E. y Vera, D. (2017). Transición de poder y orden mundial: el ascenso global de China y su proyección. En E. Pastrana Buelvas y H. Gehring (Eds.), La proyección de China en América Latina y el Caribe (pp. 23-72). Bogotá: Pontificia Universidad Javeriana.

Rodríguez Sabiote, C., Lorenzo Quiles, O. y Herrera Torres, L. (2005). Teoría y práctica del análisis de datos cualitativos. Proceso general y criterios de calidad. Revista Internacional de Ciencias Sociales y Humanidades, vol. 15, no. 2, pp. 133-154.

Roy, D. (1996). The China Threat. Asian Survey, vol. 36, no. 8, pp. 758-77. https://doi. org/10.2307/2645437

Roy, D. (1997). The Foreign Policy of Great-Power China. Contemporary Southeast Asia, vol. 19, no. 2, pp. 121-35. Recuperado el 8 de junio de 2020, de http://www.jstor.org/ stable/25798373

Salama, P. (2017). Brasil y China: caminos de fortalezas y desconciertos. Problemas del Desarrollo, vol. 48, no. 188, pp. 9-28. http://dx.doi.org/10.1016/j.rpd.2017.01.002

Samaja, J. (1996). Epistemología y Metodología. Elementos para una Teoría de la Investigación Científica. Buenos Aires: Eudeba.

Sanz, J. (2013). La influencia de China en Latinoamérica: El Consenso de Washington y el de Beijing. Cuadernos de Pensamiento Político, no. 37, pp. 145-166. Recuperado el 8 de junio de 2020, de http://www.jstor.org/stable/23511315

Shih, C-Y. (2005). Breeding a Reluctant Dragon: Can China Rise into Partnership and Away from Antagonism?. Review of International Studies, vol. 31, no. 4, pp. 755-774. 
Sinclair, J. (1991). Corpus Concordance Collocation. Oxford: Oxford University Press.

Stoddart, M. C. J. (2007). Ideology, Hegemony, Discourse: A Critical Review of Theories of Knowledge and Power. Social Thought \& Research, vol. 28, pp. 191-225. http://dx.doi. org/10.17161/STR.1808.5226

Strauss, J. C. y Armony, A.C. (2012). From the Great Wall to the New World: China and Latin America in the 21st Century. Cambridge: Cambridge University Press.

Terán Samanamud, G. (2014). China en América Latina: los casos de Ecuador y Perú entre los años 2009-2012, ¿ es posible una apuesta hacia el futuro? Anuario Mexicano de Derecho Internacional, no. 14, pp. 221-260. http://dx.doi.org/10.1016/S1870-4654(14)70007-8

Vitelli, Marina (2014). Veinte años de constructivismo en Relaciones Internacionales. Del debate metateórico al desarrollo de investigaciones empíricas. Una perspectiva sin un marco de política exterior. PostData, vol. 19, no. 1, pp. 129-162.

Wintgens, S. (2018). La Nueva Ruta de la Seda: ¿qué rol para América Latina y el Caribe?. Puentes, vol. 19, no. 8, pp. 27-30.

Woods, A., Fletcher, P. y Hughes, A. (1991). Statistics in Language Studies. Cambridge: Cambridge University Press.

Xi, J. (2014). La Gobernación y Administración de China. Beijing: Ediciones en Lenguas Extranjeras Cía. Ltda.

Yaolin Wang, M. (2002). The Motivations behind China's Government-Initiated Industrial Investments Overseas. Pacific Affairs, vol. 75, no. 2, pp. 187-206. https://doi. org/10.2307/4127182

Yong, Z. y Peiran, S. (2018). Expanding the Belt and Road: A New Perspective on ChinaLatin America Integrated Cooperation. China Belt and Road Initiative Journal, vol. 1, no. 2, pp. 65-116.
La diplomacia china hacia América Latina: bases cooperativas para la iniciativa de la Franja y la Ruta

Mariano Mosquera 